University of Nebraska - Lincoln

DigitalCommons@University of Nebraska - Lincoln

Norman R. Simon Papers

Research Papers in Physics and Astronomy

10-1-1995

\title{
LONG-PERIOD CEPHEIDS: MODELS AND OBSERVATIONS
}

Norman R. Simon

University of Nebraska - Lincoln, nsimon@unl.edu

Shashi M. Kanbur

University of Nebraska-Lincoln, kanbur@oswego.edu

Follow this and additional works at: https://digitalcommons.unl.edu/physicssimon

Simon, Norman R. and Kanbur, Shashi M., "LONG-PERIOD CEPHEIDS: MODELS AND OBSERVATIONS" (1995). Norman R. Simon Papers. 44.

https://digitalcommons.unl.edu/physicssimon/44

This Article is brought to you for free and open access by the Research Papers in Physics and Astronomy at DigitalCommons@University of Nebraska - Lincoln. It has been accepted for inclusion in Norman R. Simon Papers by an authorized administrator of DigitalCommons@University of Nebraska - Lincoln. 


\title{
LONG-PERIOD CEPHEIDS: MODELS AND OBSERVATIONS
}

\author{
Norman R. Simon AND Shashi M. Kanbur ${ }^{1}$ \\ Department of Physics and Astronomy, University of Nebraska, Lincoln, NE 68588-0111 \\ Received 1995 February 13; accepted 1995 April 6
}

\begin{abstract}
We compile a list of 50 long-period Cepheids $\left(12^{\mathrm{d}}<P<70^{\mathrm{d}}\right)$ with light curves suitable for Fourier decomposition. For $P>20^{\mathrm{d}}$, the plots versus period of the Fourier quantities $\phi_{21}, \phi_{31}, \phi_{41}$, and $R_{21}$ all show a slow rise, with considerable scatter. Hydrodynamic calculations are performed to model the observed stars. The theoretical light curves are converted from bolometric to visual, and the Fourier coefficients compared with the observed ones. While the theoretical values follow the observed trends in a crude, general sense, differences are noted. At the lower end of the period range the models depart systematically from the observed stars. At longer period, the differences are much less striking, perhaps being obscured by scatter in the observations. We conclude that a detailed comparison between theory and observation must await a more extensive and accurate sample of observed stars.
\end{abstract}

Subject headings: Cepheids - hydrodynamics - stars: oscillations

\section{INTRODUCTION}

Recent observations of Cepheids in the Virgo Cluster galaxies NGC 4571 (Pierce et al. 1994) and M100 (Freedman et al. 1994) comprise a vigorous start on the final process that will nail down the Hubble constant, $H_{0}$. The Cepheids monitored in these studies had periods which ranged from 20 to $90^{\mathrm{d}}$. Indeed, the extension of the primary distance scale to Virgo and beyond will depend upon observations made at the longperiod end of the Cepheid sample. Unfortunately, it is just these long-period Cepheids which have been least studied, both observationally and theoretically.

In the present work, we construct a grid of hydodynamic pulsation models and compare their (theoretical) light curves with those of a large observational sample of Cepheids with $P>12^{\text {d }}$. The method used is that of Fourier decomposition, where the light curves are fitted with a Fourier series, $A_{0}+A_{j}$ $\cos \left(j w t+\phi_{j}\right)$ [summation convention], and their shapes quantified in terms of combinations of the low-order coefficients, viz., $\phi_{j 1}=\phi_{j}-j \phi_{1}$ and $R_{j 1}=A_{j} / A_{1}$. We shall find that while the models crudely duplicate the observed runs of the Fourier coefficients, neither the calculations nor the observations are at present precise enough to determine fundamental parameters for the observed stars.

\section{THE OBSERVATIONS}

Table 1 lists in order of increasing period 50 Cepheids with $P>12^{\text {d }}$. The first six columns give the star name and period, followed by the values of the Fourier quantities $R_{21}, \phi_{21}, \phi_{31}$, and $\phi_{41}$ as determined by Fourier decomposition (Simon \& Lee 1981) of the $V$-magnitude light curves. The last column provides literature references as indicated in the footnote. The Pel data were converted from the Walraven to the Johnson system (using the prescription given by Pel 1976) before the Fourier technique was applied. The Cepheids are all galactic, except for five stars taken from the Saha et al. (1994) observations of IC 4182, the galaxy which contains the historical

\footnotetext{
${ }^{1}$ Current address: Department of Physics and Astronomy, University of Glasgow, Glasgow G12 8QQ, Scotland.
}

Type Ia supernova, 1937C. The Table 1 sample consists of all stars in the cited references whose light curves were deemed suitable for Fourier decomposition (see, e.g., Simon \& Lee 1981; Simon \& Moffett 1985).

In Figures 1-4, we plot versus period the Fourier terms $\phi_{21}$, $\phi_{31}, \phi_{41}$, and $R_{21}$, respectively. The different symbols denote different observers as indicated in the captions. No set of observations stands out as distinct in these diagrams; this includes the data for the Cepheids in IC 4182 (see also Simon \& Clement 1994). The sharp rise at short period in all the Fourier terms is due to the Population I Cepheid resonance near $10^{\mathrm{d}}$ (Simon \& Schmidt 1976; Simon \& Lee 1981). Beyond $20^{d}$, there is a slower rise with considerably more scatter. This is generally true of all the Fourier terms, except that the two stars of longest period break this trend by showing relatively small values of $R_{21}$ (Fig. 4).

A number of correlations may be noted among the Fourier terms. Figures 5, 6, and 7 display plots of $\phi_{41}$ versus $\phi_{31}, \phi_{31}$ versus $R_{21}$, and $\phi_{41}$ versus $R_{21}$, respectively. In all three plots, the quantities are seen to increase in tandem. This agrees with correlations found between $\phi_{31}$ and $R_{21}$ among the RRc stars in $\omega$ Centauri (Simon 1990a), and between $\phi_{41}$ and $\phi_{31}$ in a group of galactic Cepheids of shorter period (Simon \& Moffett 1985). In Figure 5, there are seven stars which stand out from the main trend. The five points which are below and the two above may be made to conform by increasing their values of $\phi_{41}$ in the former case and decreasing them in the latter. The same changes applied to these seven stars in the $\phi_{41}$ versus. $R_{21}$ diagram (Fig. 7) are then seen to reduce the scatter in that figure significantly. Since the fourth-order Fourier term has the smallest amplitude of any treated here and is thus the most difficult to determine from the fit, it is not surprising that $\phi_{41}$ may be in error for a number of stars. This analysis hints that the trends in Figures 5-7 (and perhaps those in Figs. 1-4) may be considerably tighter than they appear in the present data.

\section{THE MODELS}

We constructed 33 hydrodynamic pulsation models using the code TGRID (Simon \& Aikawa 1986) with OPAL opacities. The chemical composition was $X=0.70, Z=0.02$ and 
SIMON \& KANBUR

TABLE 1

Fourier Decomposition Parameters for LoNG-Period Cepheids

\begin{tabular}{|c|c|c|c|c|c|c|}
\hline Star & Period & $R_{21}$ & $\phi_{21}$ & $\phi_{31}$ & $\phi_{41}$ & Reference \\
\hline KK Cen & 12.180 & 0.243 & 4.196 & 7.158 & 4.848 & $\mathbf{P}$ \\
\hline vX Cru $\ldots \ldots \ldots$ & 12.213 & 0.306 & 4.166 & 7.899 & 4.477 & $\mathbf{P}$ \\
\hline SS CMa .......... & 12.362 & 0.168 & 4.171 & 6.925 & 4.072 & $\mathbf{P}$ \\
\hline U Nor...$\ldots \ldots \ldots$ & 12.641 & 0.179 & 4.211 & 7.300 & 4.461 & $\mathbf{P}$ \\
\hline SY Nor $\ldots \ldots \ldots$ & 12.645 & 0.166 & 4.183 & 7.277 & 5.272 & $\mathbf{P}$ \\
\hline SU Cru ......... & 12.848 & 0.252 & 3.806 & 7.593 & 5.875 & $\mathbf{P}$ \\
\hline Z Sct & 12.901 & 0.166 & 4.604 & 7.572 & 5.262 & MB \\
\hline EX Vel ... & 13.234 & 0.056 & 3.617 & 6.490 & 4.593 & $\mathbf{P}$ \\
\hline FI Car .......... & 13.454 & 0.010 & 4.199 & 6.711 & 4.675 & $\mathbf{P}$ \\
\hline VY Sgr $\ldots \ldots \ldots \ldots$ & 13.557 & 0.239 & 4.460 & 7.720 & 5.700 & $\mathbf{P}$ \\
\hline BN Pup ........... & 13.673 & 0.243 & 4.750 & 8.458 & 6.028 & $\mathbf{P}$ \\
\hline SZ Cas ........... & 13.638 & 0.212 & 4.012 & 8.254 & 5.132 & MB \\
\hline TT Aql ........... & 13.755 & 0.226 & 4.510 & 7.855 & 5.678 & $\mathbf{P}$ \\
\hline TX Cyg ......... & 14.710 & 0.254 & 4.417 & 7.978 & 5.796 & MB \\
\hline UZ Sct ........... & 14.744 & 0.159 & 4.234 & 7.436 & 5.604 & $\mathbf{P}$ \\
\hline RW Cas......... & 14.792 & 0.223 & 4.549 & 7.762 & 5.803 & MB \\
\hline SZ Cyg $\ldots \ldots \ldots \ldots$ & 15.110 & 0.218 & 4.259 & 7.713 & 5.732 & MB \\
\hline SV Mon .......... & 15.233 & 0.240 & 4.585 & 8.019 & 5.972 & MB \\
\hline X Cyg $\ldots \ldots \ldots \ldots$ & 16.386 & 0.244 & 4.433 & 8.050 & 5.958 & MB \\
\hline RW Cam ......... & 16.415 & 0.227 & 4.336 & 7.716 & 5.538 & MB \\
\hline CD Cyg $\ldots \ldots \ldots$ & 17.074 & 0.270 & 4.306 & 8.295 & 6.226 & MB \\
\hline TX Cen .......... & 17.094 & 0.253 & 4.404 & 8.054 & 5.968 & $\mathbf{P}$ \\
\hline Y Oph .......... & 17.127 & 0.223 & 4.002 & 7.681 & 3.974 & MB \\
\hline SZ Aql $\ldots \ldots \ldots \ldots$ & 17.138 & 0.266 & 4.415 & 8.078 & 5.967 & $\mathbf{P}$ \\
\hline C4-V10.......... & 18.3 & 0.315 & 4.37 & 8.03 & 6.36 & $\mathbf{S}$ \\
\hline VY Car .......... & 18.921 & 0.271 & 4.468 & 8.042 & 6.165 & $\mathbf{P}$ \\
\hline RU Sct ......... & 19.704 & 0.316 & 4.381 & 8.517 & 6.580 & $\mathbf{P}$ \\
\hline RY Sco $\ldots \ldots \ldots$ & 20.316 & 0.320 & 4.086 & 8.524 & 6.705 & $\mathbf{P}$ \\
\hline WZ Sgr .......... & 21.850 & 0.299 & 4.545 & 8.336 & 6.428 & MB \\
\hline WZCar .......... & 23.021 & 0.407 & 4.580 & 9.087 & 7.423 & $\mathbf{P}$ \\
\hline VZ Pup .......... & 23.164 & 0.402 & 4.460 & 8.906 & 7.234 & $\mathbf{P}$ \\
\hline $\mathrm{C} 1-\mathrm{V} 4 \ldots \ldots \ldots$ & 24.7 & 0.329 & 4.60 & 8.74 & 7.25 & $\mathbf{S}$ \\
\hline X Pup $\ldots \ldots \ldots \ldots$ & 25.961 & 0.411 & 4.512 & 9.318 & 7.576 & $\mathbf{P}$ \\
\hline T Mon ........... & 27.021 & 0.344 & 4.511 & 8.809 & 7.005 & $\mathbf{P}$ \\
\hline RY Vel .......... & 28.127 & 0.387 & 4.444 & 9.120 & 7.336 & $\mathbf{P}$ \\
\hline V609 Cyg ........ & 31.076 & 0.440 & 4.565 & 9.252 & 7.695 & B \\
\hline V396 Cyg ........ & 33.247 & 0.342 & 4.453 & 8.698 & 6.924 & B \\
\hline KN Cen ......... & 34.019 & 0.405 & 4.475 & 8.848 & 7.048 & $\mathbf{P}$ \\
\hline $\mathrm{C} 4-\mathrm{V} 8 \ldots \ldots \ldots$ & 35.2 & 0.395 & 4.78 & 9.37 & 8.59 & $\mathbf{S}$ \\
\hline L Car .... & 35.533 & 0.315 & 4.400 & 8.641 & 6.956 & $\mathbf{P}$ \\
\hline $\mathrm{C} 2-\mathrm{V} 2 \ldots \ldots \ldots$ & 37.5 & 0.439 & 4.93 & 9.66 & 8.45 & $\mathrm{~S}$ \\
\hline EV Aql $\ldots \ldots \ldots \ldots$ & 38.629 & 0.374 & 4.658 & 9.388 & 7.580 & B \\
\hline $\mathrm{U}$ Car ............... & 38.768 & 0.459 & 4.800 & 9.570 & 7.669 & $\mathbf{P}$ \\
\hline RS Pup $\ldots \ldots \ldots$ & 41.387 & 0.377 & 4.852 & 9.436 & 7.823 & $\mathbf{P}$ \\
\hline $\mathrm{C} 1-\mathrm{V} 6 \ldots \ldots \ldots$ & 42.0 & 0.432 & 4.70 & 9.62 & 7.94 & $\mathrm{~S}$ \\
\hline SV Vul. & 45.024 & 0.426 & 4.667 & 9.379 & 7.664 & B \\
\hline V1467 Cyg....... & 48.524 & 0.426 & 4.881 & 9.672 & 8.070 & B \\
\hline CE Pup .......... & 49.530 & 0.397 & 5.088 & 9.478 & 9.029 & $\mathbf{P}$ \\
\hline GY Sge $\ldots \ldots \ldots$ & 51.601 & 0.326 & 4.763 & 9.578 & 7.665 & B \\
\hline S Vul. & 68.003 & 0.291 & 4.952 & 9.778 & 8.290 & B \\
\hline
\end{tabular}

REFerENCES. $-\mathrm{P}=$ Pel 1976; MB $=$ Moffett \& Barnes 1985; $=$ Saha et al. 1994;
B = Berdnikov 1994.

convection was neglected. The model parameters had ranges: $5.5 \leq M \leq 12.0 M_{\odot}, 3.597 \leq \log L \leq 4.534$, and $5000 \leq T_{e} \leq$ '5600. The bulk of these models followed the evolutionary relation (Chiosi 1989)

$$
\log L=3.61 \log M+0.94 \text {, }
$$

but a few were also calculated according to (Becker, Iben, $\&$ Tuggle 1977)

$$
\log \mathrm{L}=3.68 \log M+0.46 .
$$

Although more recent evolutionary tracks produce relations which differ somewhat from equations (1) and (2), we shall argue below that the Fourier coefficients of the theoretical light curves will be insensitive to such differences within the uncertainty caused by imprecision in the observations.
The bolometric light variations produced by TGRID were converted to visual magnitudes by employing a bolometric correction at each point on the light curve. These corrections came from Table 1 of Kurucz (1991). To determine the bolometric corrections as a function of temperature and gravity, we fit the Kurucz data in the range $3750 \leq T_{e} \leq 8000 \mathrm{~K}, 0 \leq \log$ $g \leq 5$ with the following expression

$$
\begin{aligned}
\mathrm{BC}=A_{0}+A_{1} \log T_{e}+A_{2}( & \left.\log T_{e}\right)^{2} \\
& +A_{3}\left(\log T_{e}\right)^{3}+A_{4} \log g,
\end{aligned}
$$

where $A_{0}=-2427.959, A_{1}=1868.993, A_{2}=-479.5952$, $A_{3}=41.02427$, and $A_{4}=0.01523420$. The standard deviation of this fit is $0.023 \mathrm{mag}$, and in the range $4000 \leq T_{e} \leq 8000 \mathrm{~K}$, equation (3) never departs from the tabulated values by more 


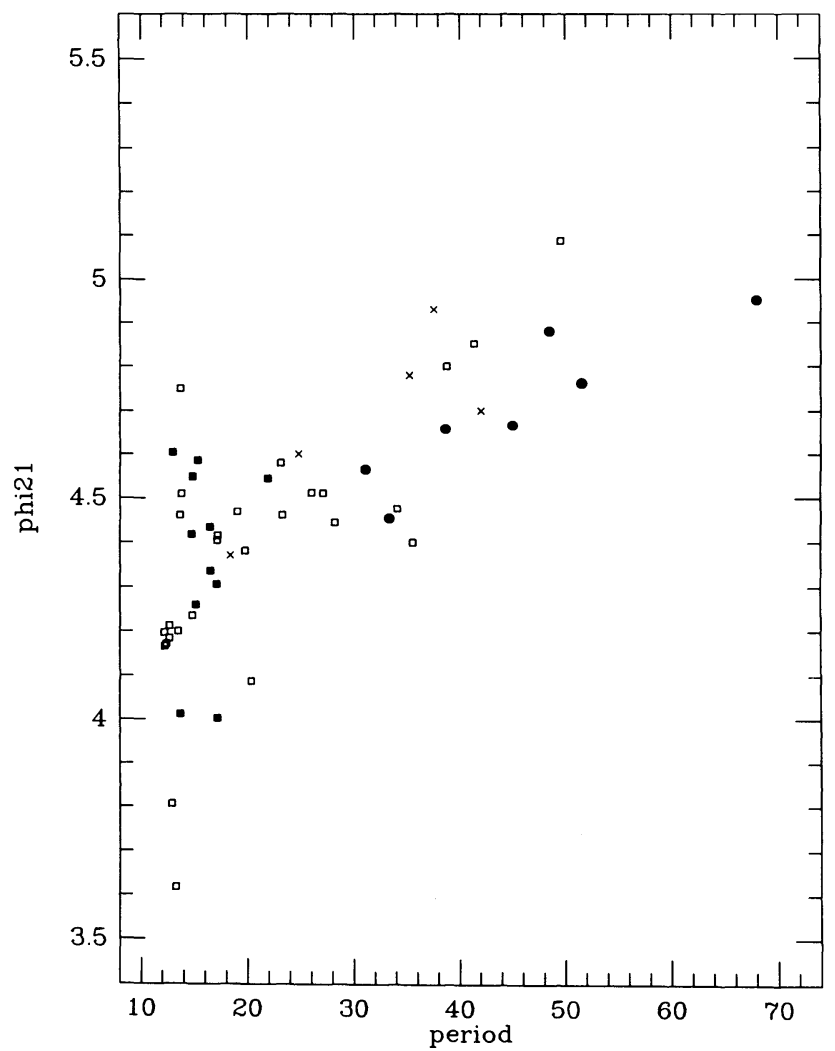

Fig. 1. $-\phi_{21}$ vs. period for observed stars. Symbols correspond to references in Table 1 as follows: open squares: $\mathrm{P}$; filled squares: $\mathrm{MB}$; dots: $B$; crosses: $\mathrm{S}$.

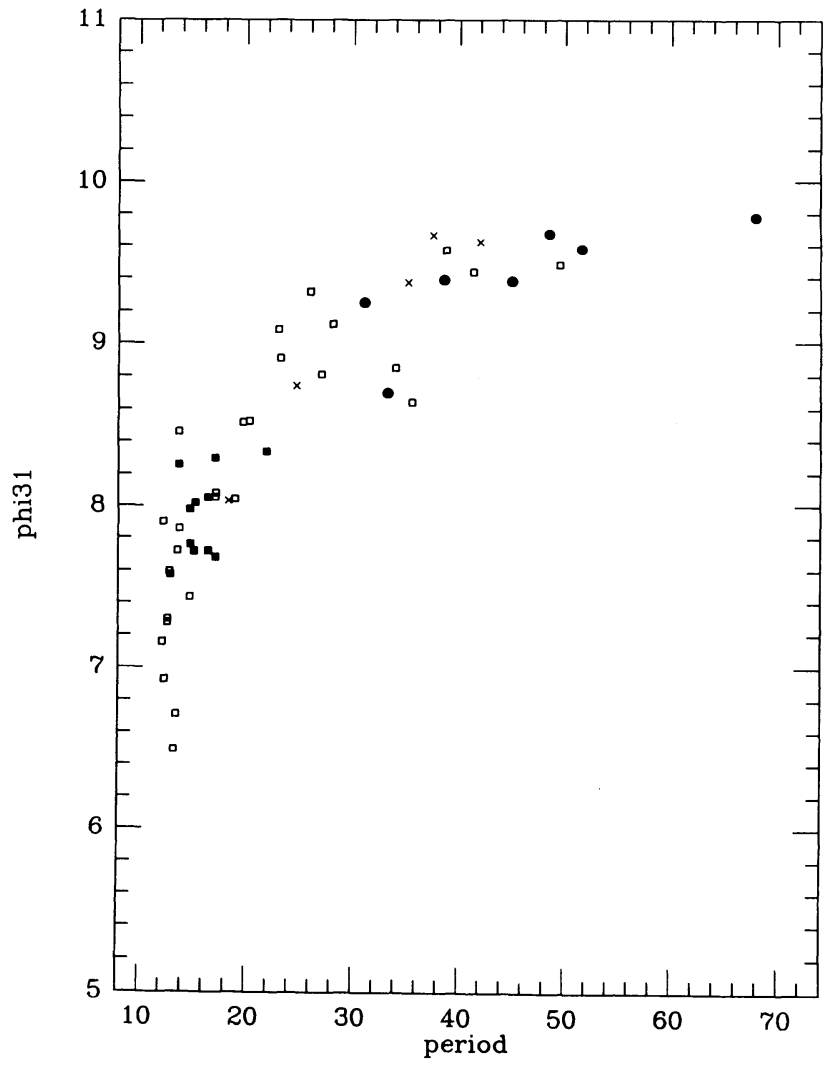

FIG. $2 .-\phi_{31}$ vs. period for observed stars. Symbols as in Fig. 1

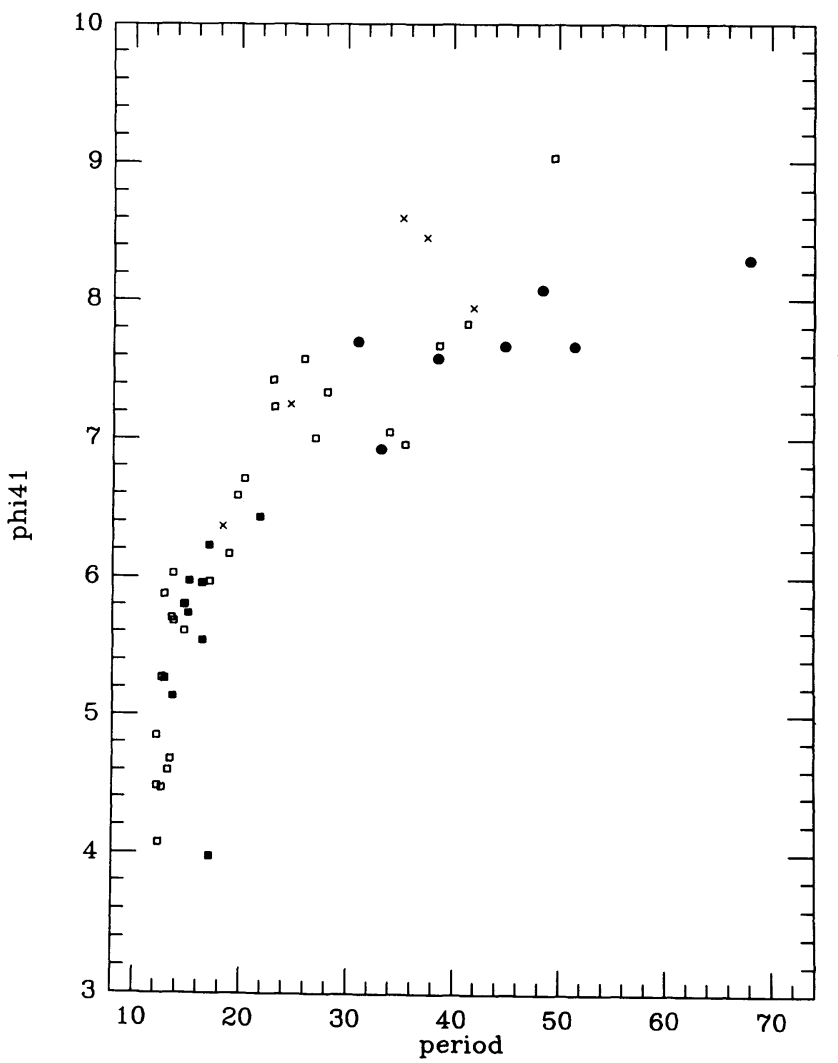

FIG. $3 .-\phi_{41}$ vs. period for observed stars. Symbols as in Fig. 1

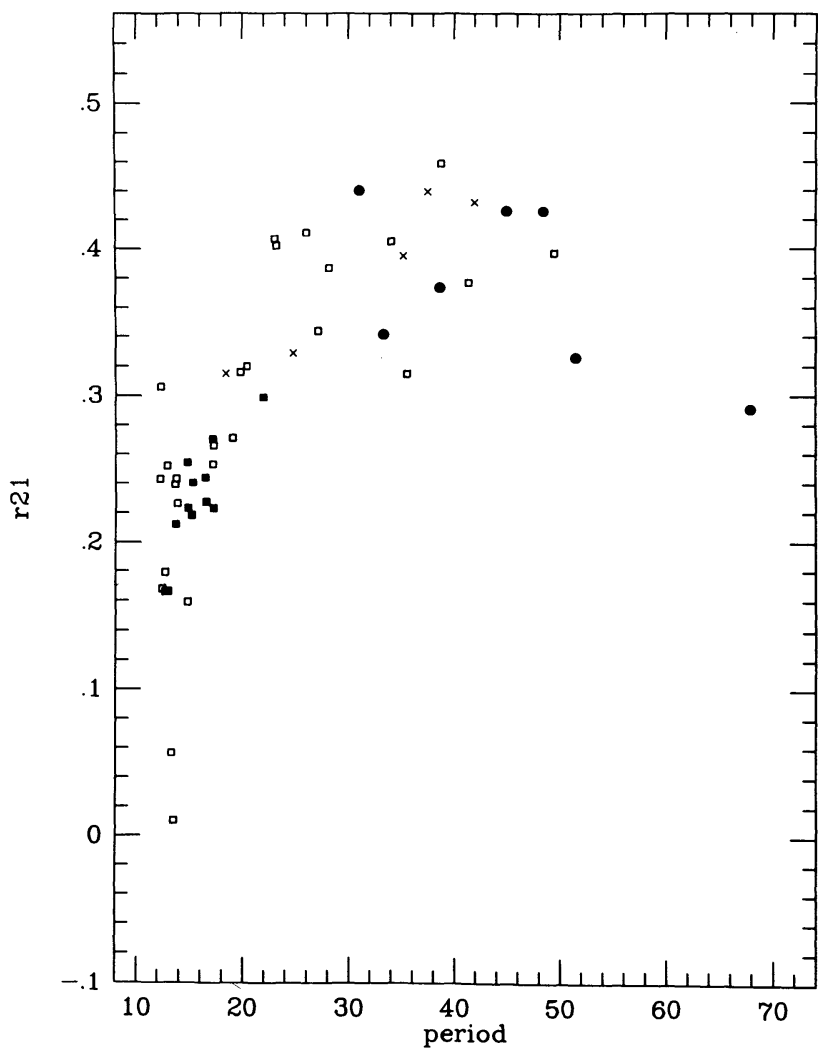

FIG. 4. $-\mathbf{R}_{21}$ vs. period for observed stars. Symbols as in Fig. 1 


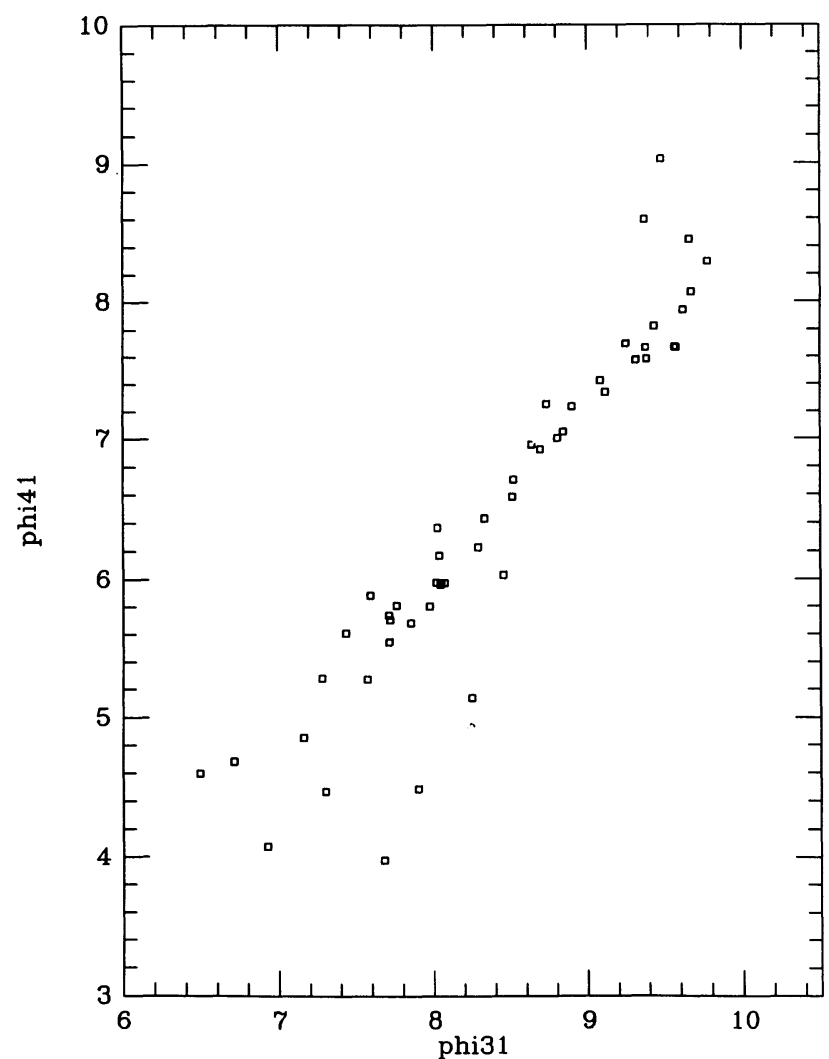

FIG. 5. $-\phi_{41}$ vs. $\phi_{31}$ for observed stars

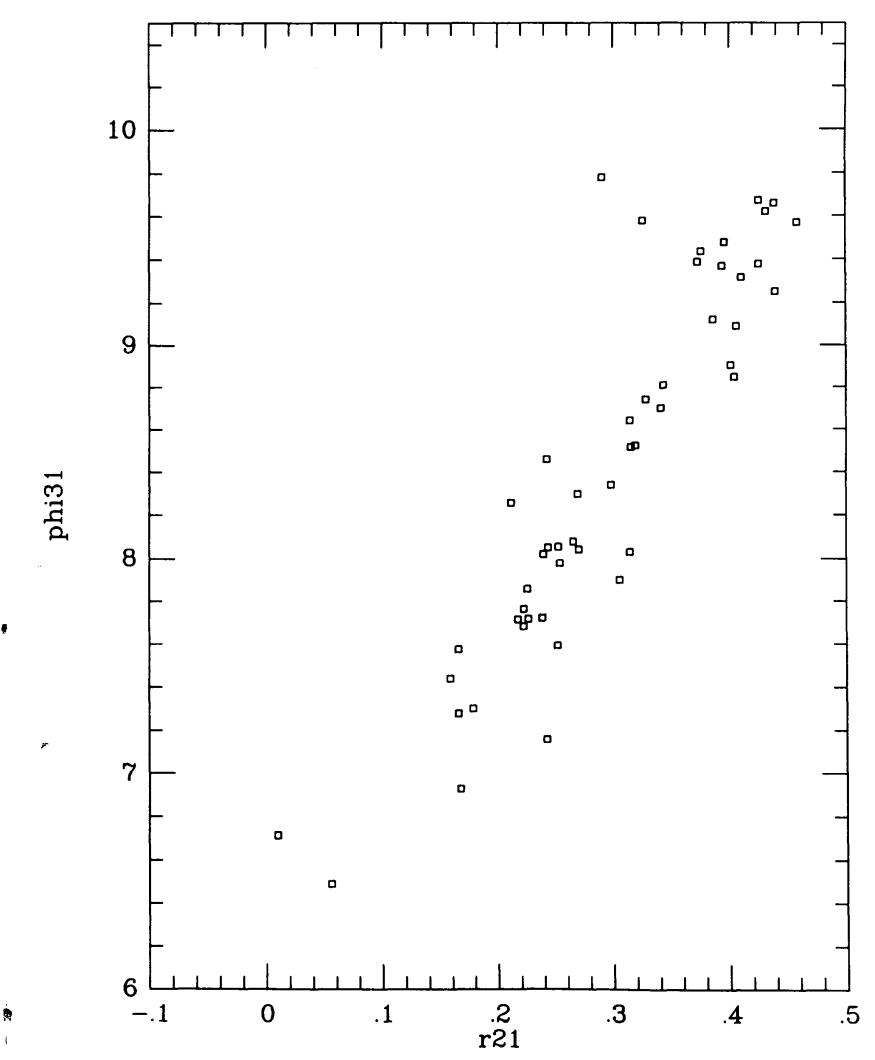

Fig. 6. $-\phi_{31}$ vs. $R_{21}$ for observed stars

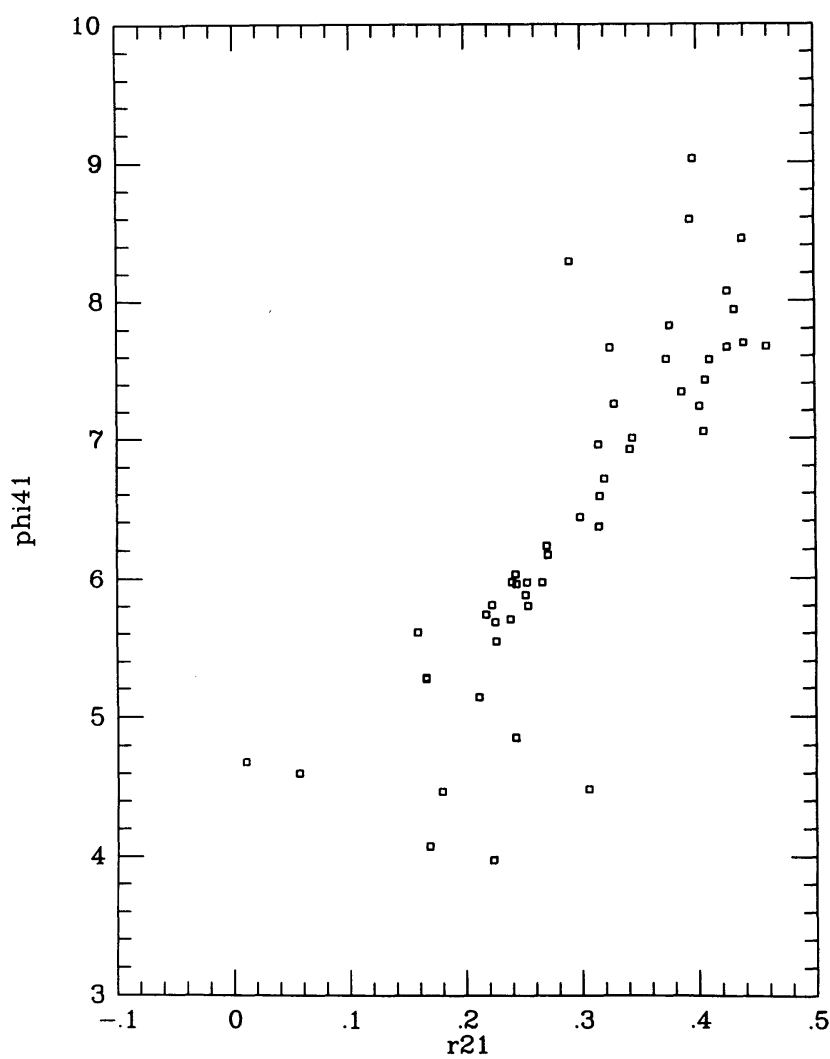

Fig. 7. $-\phi_{41}$ vs. $R_{21}$ for observed stars

than 0.04 mag. This expression should not be used for temperatures below $4000 \mathrm{~K}$ or above $8000 \mathrm{~K}$.

With the theoretical light curves, visual and bolometric, thus in hand, we subjected both sets to Fourier decomposition. For almost all models, the visual Fourier terms had values slightly lower than the bolometric. The average differences, bolometric minus visual, were $0.11,0.19,0.24$, and 0.015 for $\phi_{21}, \phi_{31}, \phi_{41}$, and $R_{21}$, respectively. These differences are of the same size but opposite sign to those given for a small number of cases by Simon \& Moffett (1985). We beleve that the Simon-Moffett values were reported with incorrect sign.

Table 2 presents the model parameters along with the visual Fourier decomposition coefficients. In Figures 8-11 we plot versus period the runs of $\phi_{21}, \phi_{31}, \phi_{41}$, and $R_{21}$, respectively, for the hydrodynamic visual light curves. The open squares represent the models constructed according to equation (1) and the filled squares according to equation (2). The point near $14^{\mathrm{d}}$ occupies a unique position owing to the fact that the corresponding model happens to lie very close to the resonance $P_{2} / P_{0}=0.5$. The remainder of the filled squares do not depart dramatically from the open squares, except perhaps in Figure 11 , where the $R_{21}$ values corresponding to equation (2) seem generally smaller. In plots of $\phi_{41}$ versus $\phi_{31}, \phi_{31}$ versus $R_{21}$, and $\phi_{41}$ versus $R_{21}$ (none of them shown here), the two sets of models turn out to be virtually indistinguishable. In any event, given the scatter in the observational quantities (Figs. 1-4), we did not deem it useful to pursue such differences further. Recent evolutionary tracks indicate a slope of about 4.0 in the luminosity-mass relation, and a zero point perhaps somewhere in between those of equations (1) and (2) (Simon 1995). Once again, given the scatter in the observed data, the differences in 
TABLE 2

Hydrodynamic Model Parameters

\begin{tabular}{cccccccc}
\hline \hline$M$ & $\log L$ & $T_{\boldsymbol{e}}$ & $P_{0}$ & $R_{21}$ & $\phi_{21}$ & $\phi_{31}$ & $\phi_{41}$ \\
\hline $6.03 \ldots \ldots$ & 3.741 & 5461 & 14.36 & 0.309 & 4.46 & 8.82 & 6.38 \\
$6.03 \ldots \ldots$ & 3.741 & 5161 & 17.32 & 0.430 & 4.72 & 9.56 & 7.83 \\
$6.46 \ldots \ldots$ & 3.849 & 5404 & 17.75 & 0.357 & 4.49 & 8.97 & 6.92 \\
$6.46 \ldots \ldots$ & 3.849 & 5104 & 21.66 & 0.425 & 4.88 & 9.49 & 7.85 \\
$7.42 \ldots \ldots$ & 4.067 & 5293 & 27.35 & 0.422 & 4.45 & 9.19 & 7.63 \\
$7.42 \ldots \ldots$ & 4.067 & 5043 & 32.32 & 0.413 & 4.70 & 9.38 & 8.14 \\
$8.50 \ldots \ldots$ & 3.880 & 5243 & 17.16 & 0.241 & 4.45 & 9.01 & 5.52 \\
$8.50 \ldots \ldots$ & 3.880 & 5043 & 19.59 & 0.292 & 4.63 & 9.18 & 7.56 \\
$9.50 \ldots \ldots$ & 4.058 & 5143 & 24.46 & 0.404 & 4.62 & 9.26 & 7.13 \\
$8.00 \ldots \ldots$ & 3.783 & 5300 & 14.16 & 0.045 & 5.32 & 5.76 & 4.05 \\
$6.00 \ldots \ldots$ & 3.733 & 5600 & 12.99 & 0.230 & 4.32 & 8.04 & 5.90 \\
$6.00 \ldots \ldots$ & 3.733 & 5350 & 15.22 & 0.337 & 4.51 & 9.19 & 6.95 \\
$5.50 \ldots \ldots$ & 3.597 & 5350 & 12.24 & 0.233 & 4.59 & 9.07 & 5.71 \\
$5.50 \ldots \ldots$ & 3.597 & 5120 & 14.24 & 0.301 & 4.68 & 9.32 & 7.96 \\
$6.50 \ldots \ldots$ & 3.859 & 5470 & 17.25 & 0.318 & 4.40 & 8.76 & 6.74 \\
$6.50 \ldots \ldots$ & 3.859 & 5270 & 19.68 & 0.386 & 4.64 & 9.22 & 7.32 \\
$6.40 \ldots \ldots$ & 3.834 & 5500 & 16.29 & 0.299 & 4.40 & 8.68 & 6.56 \\
$6.40 \ldots \ldots$ & 3.834 & 5300 & 18.53 & 0.369 & 4.58 & 9.18 & 7.22 \\
$6.20 \ldots \ldots$ & 3.785 & 5370 & 16.34 & 0.339 & 4.50 & 9.03 & 6.82 \\
$6.20 \ldots \ldots$ & 3.785 & 5500 & 15.04 & 0.283 & 4.41 & 8.59 & 6.45 \\
$6.70 \ldots \ldots$ & 3.906 & 5450 & 18.91 & 0.330 & 4.41 & 8.91 & 6.76 \\
$6.70 \ldots \ldots$ & 3.906 & 5250 & 21.55 & 0.384 & 4.65 & 9.18 & 7.35 \\
$7.00 \ldots \ldots$ & 3.975 & 5400 & 21.84 & 0.355 & 4.41 & 9.04 & 7.02 \\
$7.00 \ldots \ldots$ & 3.975 & 5200 & 24.92 & 0.363 & 4.59 & 9.12 & 7.63 \\
$7.20 \ldots \ldots$ & 4.019 & 5400 & 23.48 & 0.370 & 4.40 & 9.09 & 7.09 \\
$7.20 \ldots \ldots$ & 4.019 & 5200 & 26.81 & 0.403 & 4.55 & 9.23 & 7.69 \\
$8.00 \ldots \ldots$ & 4.184 & 5200 & 35.23 & 0.480 & 4.57 & 9.50 & 8.19 \\
$9.00 \ldots \ldots$ & 4.369 & 5200 & 48.25 & 0.445 & 4.75 & 9.63 & 8.08 \\
$10.0 \ldots \ldots$ & 4.534 & 5100 & 69.02 & 0.436 & 5.07 & 10.38 & 9.39 \\
$10.0 \ldots \ldots$ & 4.140 & 5000 & 30.87 & 0.412 & 4.89 & 9.41 & 7.71 \\
$9.00 \ldots \ldots$ & 4.369 & 5100 & 51.76 & 0.494 & 4.82 & 9.87 & 8.39 \\
$11.0 \ldots \ldots$ & 4.292 & 5000 & 39.79 & 0.373 & 4.65 & 9.16 & 7.75 \\
$12.0 \ldots \ldots$ & 4.431 & 5000 & 50.39 & 0.495 & 4.63 & 9.55 & 8.07 \\
\hline & & & & & & & \\
\end{tabular}

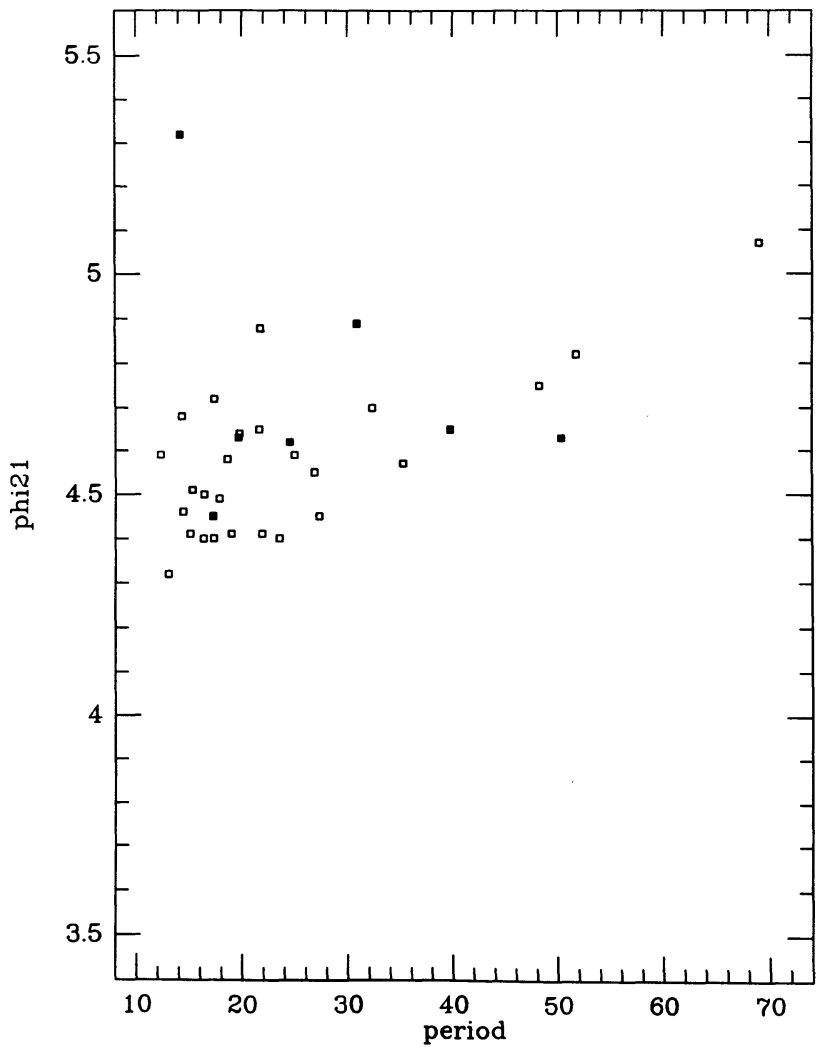

FiG. 8. $-\phi_{21}$ vs. period for models constructed according to eq. (1) (open squares) and eq. (2) (filled squares).

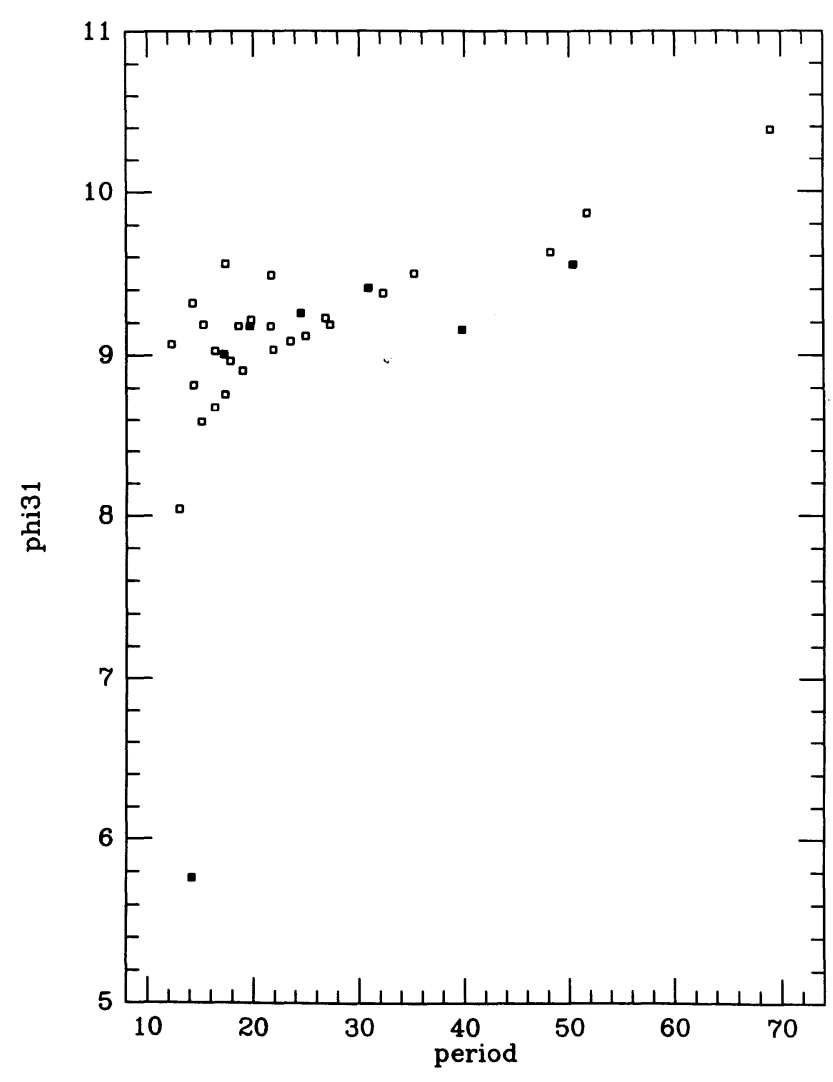

Fig. 9. $-\phi_{31}$ vs. period for models. Symbols as in Fig. 8 


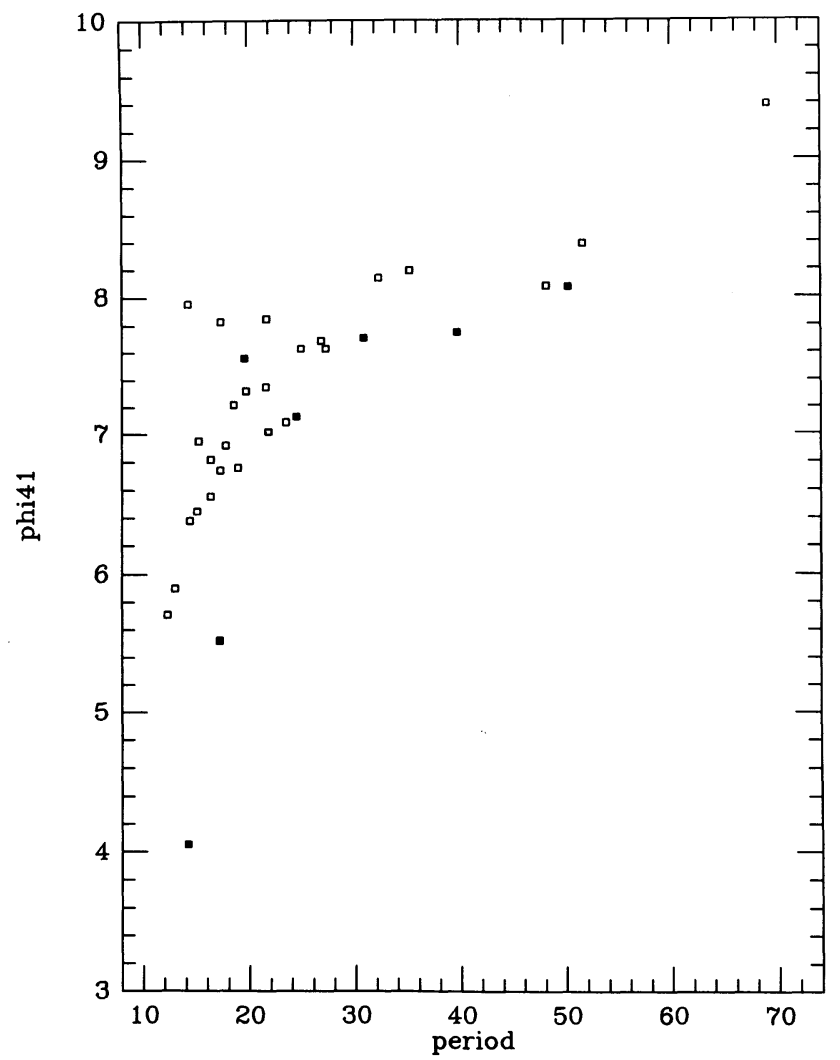

FIG. 10. $-\phi_{41}$ vs. period for models. Symbols as in Fig. 8

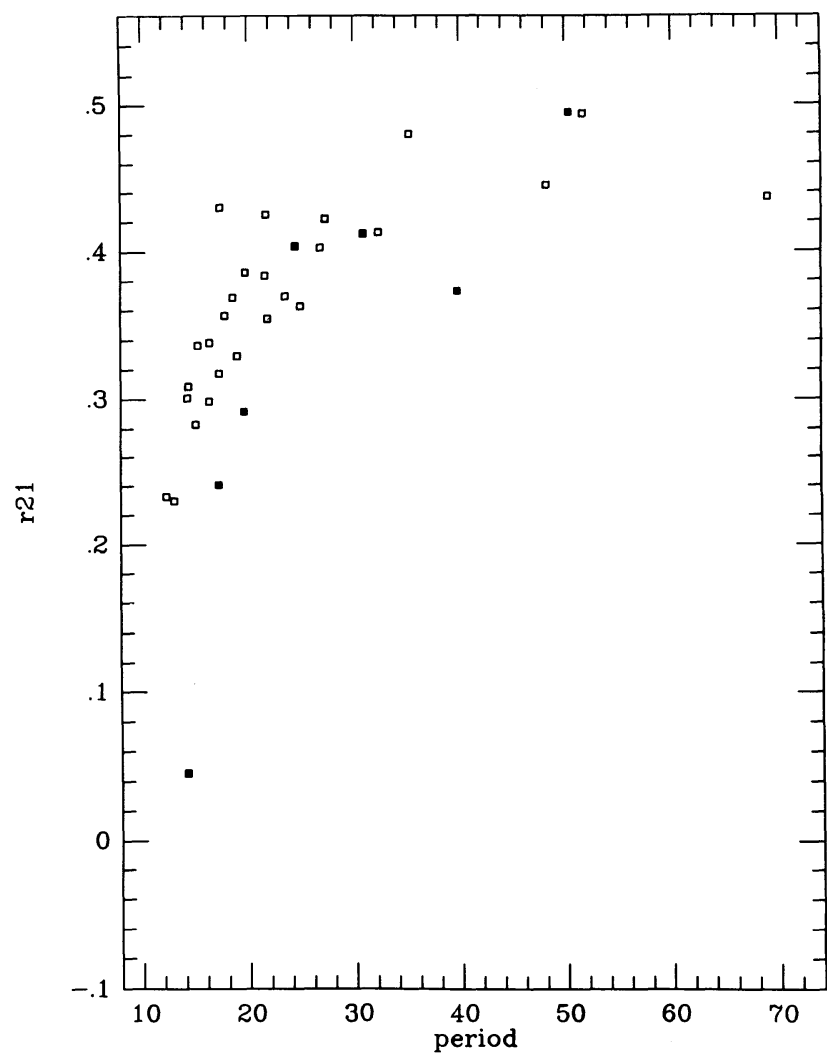

Fig. 11. $-R_{21}$ vs. period for models. Symbols as in Fig. 8 the theoretical Fourier terms occasioned by different luminosity-mass relations are not likely to be significant in any current comparison between observation and theory.

\section{THEORY VERSUS OBSERVATION}

In Figures 12-15, we once more show plots of the Fourier quantities versus period. Here the filled squares represent the models (visual magnitudes) and the open squares the observed stars. In Figure 12, the two sets generally coincide, except that the models cannot reproduce observed light curves with $\phi_{21}$ values of less than about 4.5. In the period range from about $10^{\mathrm{d}}$ to $30^{\mathrm{d}}$, the theoretical values of $\phi_{31}$ (Fig. 13) are systematically higher than those observed. At longer periods any differences are not so marked. Similar behavior may be noted in the $\phi_{41}$ plot displayed in Figure 14, while Figure 15 shows theo- retical $R_{21}$ values that tend to be larger than the observed quantities for all periods longer than about $15^{\mathrm{d}}$.

Recent studies comparing observed Cepheids with hydrodynamic pulsation models have been made by Buchler, Moskalik, \& Kovács (1990) and Moskalik, Buchler, \& Marom (1992). These studies were designed to probe the "bump Cepheid" region and hence were essentially limited to stars with $P \lesssim 20^{\mathrm{d}}$. Nonetheless, in the domain of overlap between the present investigation and the earlier work, the results are very similar (see, in particular, Fig. 6 of Moshalik et al. 1992). Since the hydrodynamic codes employed by the two groups are rather different, the similarity of the theoretical light curves is encouraging.

\section{DISCUSSION}

Figures 1-4 show general trends of slow increase of the Fourier quantities $\phi_{21}, \phi_{31}, \phi_{41}$, and $R_{21}$ in the domain $P>20^{\text {d }}$. Compared with the shorter period stars, the scatter is substantial. While there is some indication that observational error may play a large role in this scatter (see $\S 2$ above), it may also be that the Cepheids of long period constitute a more diverse group than do the shorter period stars. Such a possibility is particularly interesting in view of some difficulties in accommodating the long-period Cepheid sample within the framework of standard evolutionary tracks (Simon 1995).

Comparing the observed stars with theoretical calculations, Figures 12-15 show significant discrepancies among the shorter period objects, $P \lesssim 20^{\mathrm{d}}$. This is a reprise of well-known failings of the hydrodynamic models (e.g., Simon 1990b). Among the long-period stars, the considerable observational scatter makes the situation less clear. The models may be more successful in this domain, but a test of this must await a larger, more accurate observational sample such as that now being compiled in MACHO studies of the Large Magellanic Cloud (Cook et al. 1994). In any event, the old dream of using Fourier coefficients to precisely constrain Cepheid parameters still seems a long way from realization.

We are pleased to acknowledge support for this work under the NASA Astrophysical Theory Program, grant NAGW2395. 


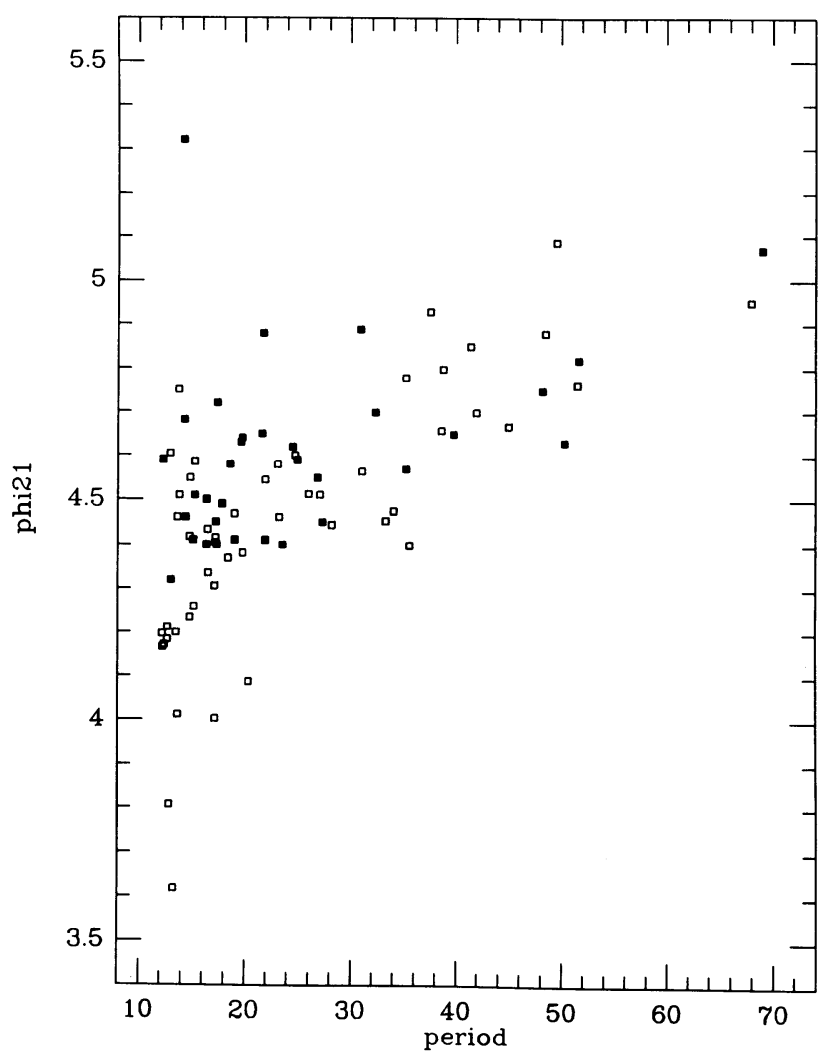

FIG. 12. $-\phi_{21}$ vs. period: open squares: observed stars; filled squares: models.

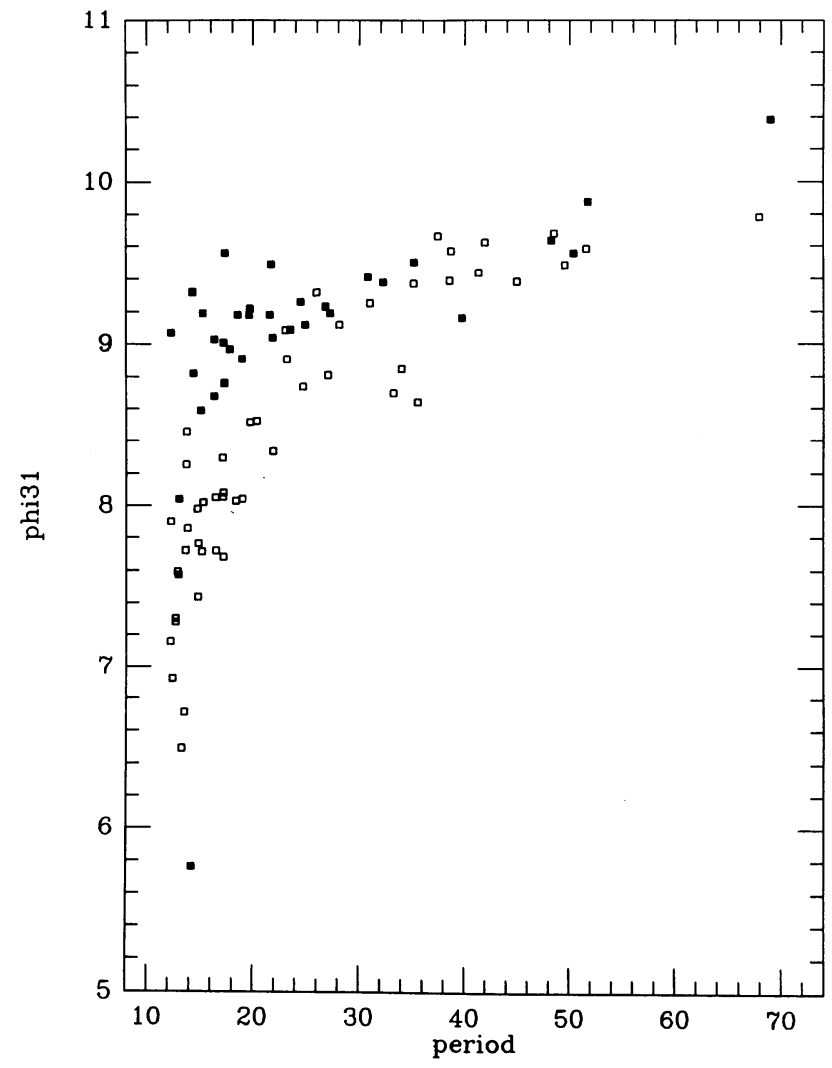

FIG. 13. $-\phi_{31}$ vs. period. Symbols as in Fig. 12

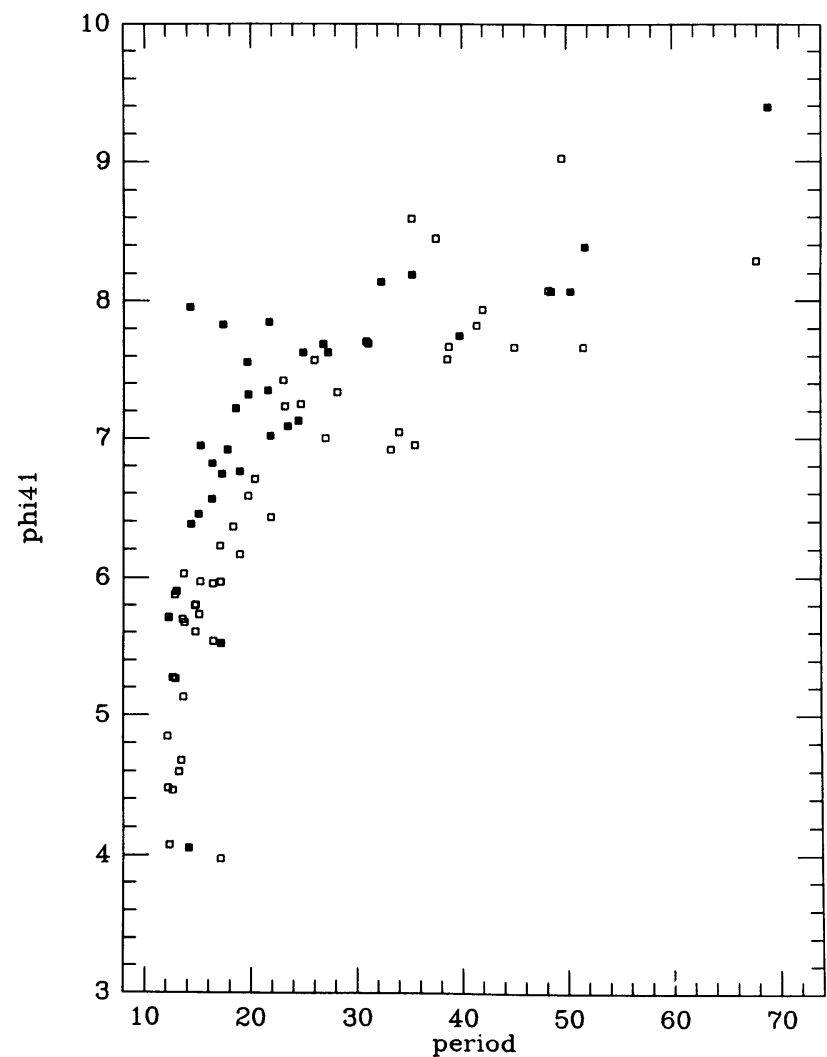

Fig. 14. $-\phi_{41}$ vs. period. Symbols as in Fig. 12

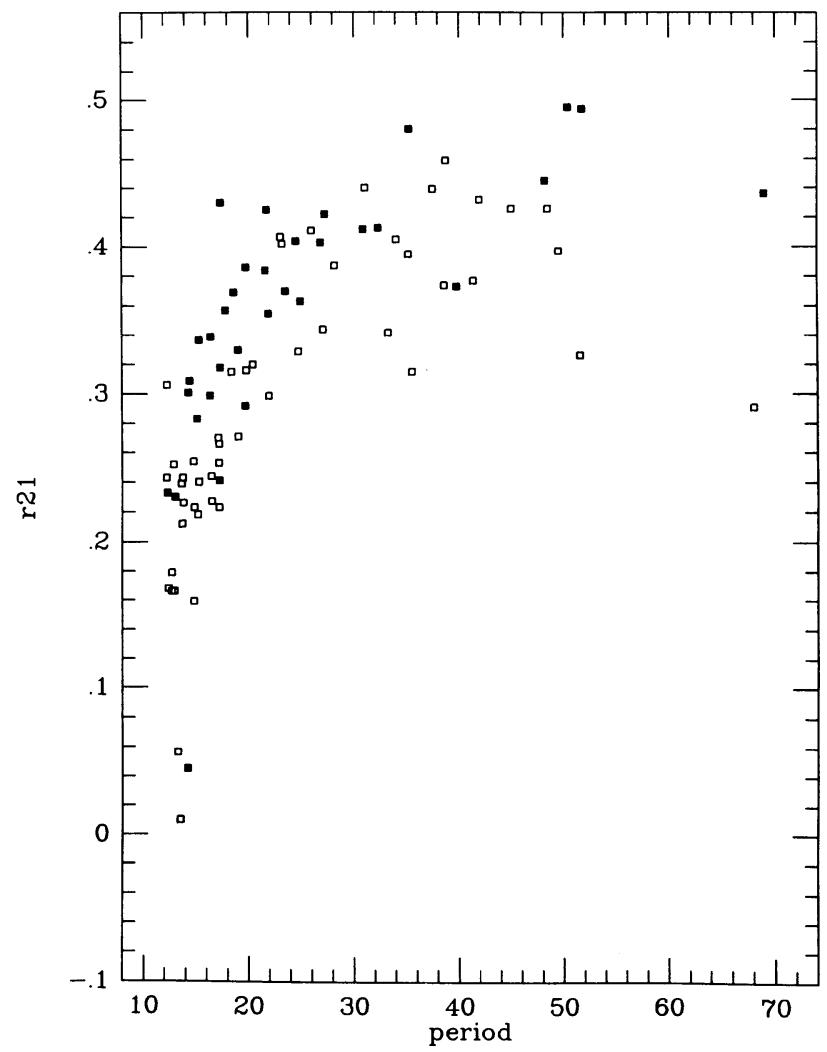

FIG. $15 .-R_{21}$ vs. period. Symbols as in Fig. 12 


\section{REFERENCES}

Becker, S. A., Iben, I., \& Tuggle, R. S. 1977, ApJ, 218, 633

Berdnikov, L. N. 1994, Soviet Astron. Lett., 20, 285

Buchler, J. R., Moskalik, P., \& Kovács, G. 1990, ApJ, 351, 617

Chiosi, C. 1989, in The Use of Pulsating Stars in Fundamental Problems of Astronomy, ed. E. G. Schmidt (Cambridge: Cambridge Univ. Press), 19

Cook, K., et al. 1994, in Astronomy Posters (Abstracts) for the 22nd General Assembly of IAU, ed. H. van Woerden (Sliedrecht: Twin Press), 138

Freedman, W. L., et al. 1994, Nature, 371, 757

Kurucz, R. L. 1991, in Precision Photometry, ed. A. G. Davis Phillip, A. R. Upgren, \& K. A. Janes (Schenectady: L. Davis Press), 27

Moffett, T. J., \& Barnes, T. G. 1985, ApJS, 58, 843

Moskalik, P., Buchler, J. R., \& Marom, A. 1992, ApJ, 385, 685

Pel, J. W. 1976, A\&AS, 24, 413
Pierce, M. J., et al. 1994, Nature, 371, 385

Saha, A., et al. 1994, ApJ, 425, 14

Simon, N. R. 1990a, MNRAS, 246, 70

$1990 \mathrm{~b}$, in Confrontation between Stellar Pulsation and Evolution, ed. C. Cacciari \& G. Clementini (ASP Conf. Ser. Vol. 11), 193 1995, in Astrophysical Applications of Powerful New Atomic Data

Bases, ed. S. J. Adelman \& W. L. Wiese (ASP Conf. Ser. Vol. 78), 211

Simon, N. R., \& Aikawa, T. 1986, ApJ, 304, 249

Simon, N. R., \& Clement, C. M. 1994, ApJ, 419, L21

Simon, N. R., \& Lee, A. S. 1981, ApJ, 248, 291

Simon, N. R., \& Moffett, T.J. 1985, PASP, 97, 1078

Simon, N. R., \& Schmidt, E. G. 1976, ApJ, 205, 162 